Z. Klin. Chem. Klin. Biochem.

11. Jg. 1973, S. $152-154$

\title{
Eine automatische kinetische Bestimmungsmethode der Cholinesterase (EC 3.1.1.8) im Serum
}

\author{
Von A. Eberhard und R. KLĖy \\ Aus dem Klinisch-Chemischen Zentrallaboratorium (Leiter: Prof. Dr. Dr. H. Greiling) \\ der Mediziniscben Fakultät der Rheinisch-Westfälischen Tecbnischen Hocbschule Aacben
}

(Eingegangen am 9. November 1972/15. Januar 1973)

Es wird eine automatische kinetische Bestimmung der Pseudocholinesterase im Serum mit dem Enzymautomaten 5010 Eppendorf beschrieben. Die Methode verwendet Acetylthiocholin als Substrat und 5:5'-Dithiobis-2-nitrobenzoesäure als Reagenz für SH-Gruppen. Nach dem Verfahren lassen sich 120 Proben/h analysieren. Die Zuverlässigkeitskriterien der Methode werden dargelegt.

\section{An automated kinetic determination method for serum cholinesterase (EC 3.1.1.8)}

An automated kinetic method is described for the determination of cholinesterase activities in sera using an automatic enzyme analyser 5010 Eppendorf. The method uses acetylthiocholine as substrate and 5:5'-dithiobis-(2-nitrobenzoic acid) as the reagent for SH-groups. With this method 120 determinations per hour can be made. The criteria of reliability are reported for the method.

Die Bestimmung der Pseudocholinesterase im Serum hat in der klinisch-chemischen Diagnostik ihren festen Platz. Im folgenden beschreiben wir eine Bestimmungsmethode der Pseudocholinesterase mit dem Enzymautomaten der Firma Eppendorf (Hamburg) Modell 5010 nach dem Prinzip von Ellman (1) und Garry (2).

\section{Material und Methoden}

Reagenzien

Lösung 1

(Puffer/Chromogen-Gemisch) $50 \mathrm{mmol} / 1$ Phosphatpuffer $\mathrm{pH} \mathrm{7,2}$ $0,25 \mathrm{mmol} / 1$ Dithiobisnitrobenzoesäure

\section{Lösung 2}

$156 \mathrm{mmol} / 1$ Acetylthiocholinjodid

Bestimmungsansatz

\begin{tabular}{|c|c|c|}
\hline & & Endkonzentration \\
\hline Puffer-Chromogenlösung 1 & $1,00 \mathrm{ml}$ & $\begin{array}{l}0,47 \mathrm{mmol} / 1 \text { Phosphat- } \\
\text { puffer }\end{array}$ \\
\hline Serum & $0,01 \mathrm{ml}$ & $\begin{array}{l}2,4 \mu \mathrm{mol} / 1 \text { Dithiobis- } \\
\text { nitrobenzoesäure }\end{array}$ \\
\hline
\end{tabular}

Mischen. $15 \mathrm{~min}$ bei $25^{\circ} \mathrm{C}$

inkubieren

\begin{tabular}{lll}
\hline Substratlösung 2 & $0,05 \mathrm{ml}$ & $\begin{array}{l}7,36 \mathrm{mmol} / 1 \text { Acetylthio- } \\
\text { cholinjodid }\end{array}$ \\
\hline
\end{tabular}

Mischen. Extinktionszunahme

bei $405 \mathrm{~nm}$ während $154 \mathrm{~s}$

im Abstand von $25,7 \mathrm{~s}$ messen

Vorbereitung des Automaten (5010 Eppendorf)

1. Programmstecker

1-Kanal GOT, GPT, LDH, ALD, HBDH (Probenfrequenz: 120/h)

2. Probenkette

etwa $100 \mu$ l Serum pro Gefäß

\section{Probenreagenzdosierer}

Vorratszylinder , 100 “ mit Lösung 1, Schalterstellung: 100

Probenzylinder ,10“ für Serum, Schalterstellung: 10

4. Startreagenzdosierer

Zylinder ,10" mit Lösung 2, Schalterstellung: 50

5. Inkubation $15 \mathrm{~min}$ bei $+25^{\circ} \mathrm{C}$

6. Photometer $\mathrm{Hg} 405 \mathrm{~nm}$

7. Transformationsstufe Taste $\mathrm{E}=0-1$

8. Analogdrucker $5 \mathrm{~cm} / \mathrm{min}$

9. Abgleich des Photometers:

Lösung 1 in Spezialküvette pipettieren und Nullpunkt am Photometer einstellen. Stufenschalter während der ganzen Messung entsprechend der Nullpunkteinstellung stehen lassen.

Berechnung

$\mathrm{Da}$ der Enzymtestauswerter für den Eppendorf Automaten 5010 nur für eine Papiergeschwindigkeit von $2 \mathrm{~cm} / \mathrm{min}$ eingerichtet ist, markieren wir den ersten Meßpunkt der 1. Probe auf dem Druckerstreifen und drehen den Transportknopf des Auswerters 2,5 Umdrehungen von Probe zu Probe. Aus dem Tangens des gemessenen Winkels ergibt sich die Aktivität der Pseudocholinesterase im Serum nach (3)

$$
\operatorname{tg} \alpha \cdot \frac{\mathrm{a} \cdot \mathrm{V} \cdot 10^{9}}{\mathrm{~b} \cdot \varepsilon \cdot \mathrm{v} \cdot \mathrm{d}}[\mu \mathrm{mol} / \mathrm{min} \cdot \mathrm{l}]=\mathrm{U} / \mathrm{l}
$$

zu

hierbei sind

$$
\operatorname{tg} \alpha \cdot 1993[\mu \mathrm{mol} / \mathrm{min} \cdot 1]=\mathrm{U} / 1
$$

$\begin{array}{lll}\text { a } & \text { Papiervorschub } & 5 \mathrm{~cm} / \mathrm{min} \\ \text { b } & \text { Papierbreite } & 20 \mathrm{~cm} \\ \text { V } & \text { Endvolumen } & 1,06 \mathrm{ml} \\ \text { v } & \text { Probevolumen } & 0,01 \mathrm{ml} \\ \varepsilon_{405} \mathrm{~nm} & \text { Thionitrobenzoesäure } & 13,3 \cdot 10^{6} \mathrm{~cm}^{2} / \mathrm{mol} \\ \text { d } & \text { Schichtdicke } & 1 \mathrm{~cm} \\ 10^{9} & \text { Multiplikator zur Umrechnung von } \mathrm{mol} / \mathrm{cm}^{3} \text { auf } \mu \mathrm{mol} / \mathrm{l} \text {. }\end{array}$

\section{Ergebnisse}

Die Zuverlässigkeit der Methode wurde an vier handelsüblichen Kontrollseren geprüft. 
Tab. 1

Richtigkeitsprüfung der Pseudocholinesterase-Bestimmung mit vier handelsüblichen Kontrollseren

\begin{tabular}{|c|c|c|c|c|c|c|}
\hline Kontrollserum & Analysenzahl & $\begin{array}{l}\text { Sollwert }\left(\bar{x}_{3}\right) \\
(U / I)\end{array}$ & $\begin{array}{c} \pm \text { 2s Bereich } \\
(U / 1)^{\circ}\end{array}$ & $\begin{array}{l}\text { gefundener Wert }\left(\bar{x}_{1}\right) \\
(U / 1)\end{array}$ & $\begin{array}{c} \pm 2 \text { s Bereich } \\
(U / 1)\end{array}$ & $\frac{\overline{\mathbf{x}}_{\text {Soll }}-\overline{\mathbf{x}}_{\text {Ist }}}{\overline{\mathbf{x}}_{\text {Soll }}} \cdot 100$ \\
\hline Precilip') & 85 & 2250 & $1913-2588$ & 2124 & $2228-2020$ & $-5 \%$ \\
\hline Seronorm²) & 56 & 3020 & nicht angegeben & 3144 & $3314-2974$ & $+4 \%$ \\
\hline Monitrol $\left.{ }^{3}\right)$ I & 56 & 2450 & $2200-2700$ & 2309 & $2487-2131$ & $-5 \%$ \\
\hline Monitrol's II & 56 & 1810 & $1620-2000$ & 1622 & $1698-1548$ & $-10 \%$ \\
\hline
\end{tabular}

1) Fa. Boehringer, Mannheim, Kontr. No. 101

2) Fa. Molter, Heidelberg, Batch No. 117

3) Fa. Merz + Dade, München 50, Lot No. 27 A und 114 A, B

) Von den Herstellerfirmen angegebener Vertrauensbereich

\section{Linearität}

Zur Prüfung der Linearität wurde von einem Kontrollserum (Precilip Lt. Nr. 101, Fa. Boehringer (Mannheim)) eine Verdünnungsreihe hergestellt. Beim Vergleich von Ist- und Sollwerten zeigte sich eine nahezu ideale Ubereinstimmung im Bereich zwischen 35 und 4500 U/l Pseudocholinesterase im Serum (s. Abb. 1).

\section{Richtigkeit}

Das Ergebnis der Richtigkeitskontrolle zeigt die Tabelle 1.

\section{Präzision}

Das Ergebnis der Präzisionsprüfung zeigen die $\mathrm{Ta}$ bellen 2 und 3 .

\section{Driftkontrolle}

Zur Prüfung wurden zu Anfang und am Ende einer jeden Analysenserie 25 Tage lang ein Segment mit 10 Kontrollseren eingefügt. Es wurde keine Drift festgestellt. Die Anheizzeit des Gerätes (etwa $30 \mathrm{~min}$ ) muß streng eingehalten werden.

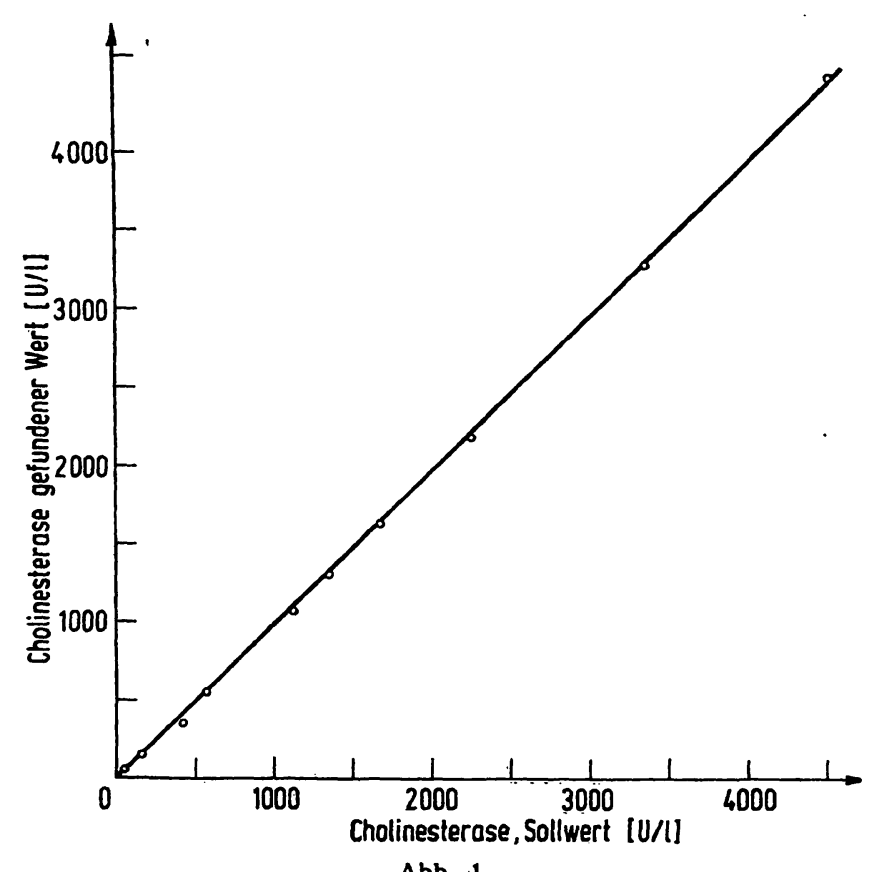

Wiederfindung der eingesetzten Pseudocholinesteraseaktivität (Verdünnungsreihe von dem Kontrollserum Precilip) durch den Enzymautomaten 5010 Eppendorf. Auf der Abszisse sind die eingesetzten und auf der Ordinate die gemessenen Enzymaktivitäten eingezeichnet. Die Gleichung der Regressionsgraden lautet:

$$
y=0,996 x+14,283 . r=0,999
$$

Tab. 2

Prăzision der Pseudocholinesterase-Bestimmung in der Serie, geprüft an vier handelsüblichen Kontrollseren

\begin{tabular}{lcccc}
\hline & $\begin{array}{c}\text { Analysen- } \\
\text { 2ahl } \\
\mathrm{n}\end{array}$ & $\begin{array}{c}\text { Mittel- } \\
\text { wert } \mathrm{X} \\
{[\mathrm{U} / \mathrm{I}]}\end{array}$ & $\begin{array}{c}\text { 2s-Bereich } \\
{[\mathrm{U} / 1]}\end{array}$ & {$[\%]$} \\
\hline Precilip & 31 & 2028 & \pm 94 & 2,3 \\
Seronorm & 31 & 2934 & \pm 150 & 2,5 \\
Monitrol 1 & 31 & 2192 & \pm 126 & 2,8 \\
Monitrol II & 38 & 1622 & \pm 74 & 2,2 \\
\hline
\end{tabular}

Tab. 3

Präzision der Pseudocholinesterase-Bestimmung von Tag zu Tag, geprüft 25 Tage lang an vier handelsüblichen Kontrollseren

\begin{tabular}{lccc}
\hline & $\begin{array}{c}\text { Mittelwert } \overline{\mathbf{x}} \\
{[U / 1]}\end{array}$ & $\begin{array}{c}\text { 2s-Bereich } \\
{[U / 1]}\end{array}$ & $\begin{array}{c}\text { Variationskoeffizient } \\
{[\%]}\end{array}$ \\
\hline Precilip & 2124 & \pm 104 & 2,4 \\
Seronorm & 3145 & \pm 170 & 2,7 \\
Monitrol I & 2309 & \pm 178 & 3,8 \\
Monitrol II & 1622 & \pm 84 & 2,2 \\
\hline
\end{tabular}

\section{Verschleppungsfebler}

Ähnlich wie bei der Drift-Kontrolle wurden AnalysenSegmente täglich mitgeführt, in denen Kontrollseren und Wasserproben sich abwechselten. Eine Verschleppung war nicht meßbar.

\section{Prïfung der nicht-enrymatischen Eigenbydrolyse von Acetyl-} thiocholin

Hierzu wurden täglich in bestimmten Segmentabschnitten der Analysenreihe Wasserproben eingefügt. Bei intakter Substratlösung (Lösung 2) konnten keine Extinktionsdifferenzen festgestellt werden (bei $+4^{\circ} \mathrm{C}$ ist die Lösung 2 mindestens 1 Woche haltbar).

\section{Speqifität der Metbode}

Die Methode ist spezifisch für die Pseudocholinesterasen im Serum (2). Aufgrund der Untersuchungen von Levine (4) läßt sich mit Acetylthiocholin die Acetylcholinesterase in den Erythrocyten (EC 3.1.1.7) nach Dialyse des gebildeten Farbstoffes bestimmen. Dieses ist mit unserer automatischen Methode nicht durchführbar, da die Eigenfärbung der hämolysierten Erythrocyten die Messung der Extinktionszunahme bei $405 \mathrm{~nm}$ stört. 


\section{Normaliverte}

An 125 Seren lebergesunder Patienten wurde ermittelt: $2490 \mathrm{U} / \mathrm{l}(1850-3030 \mathrm{U} / \mathrm{l})$.

\section{Diskussion}

Von einer modernen automatischen Enzymaktivitätsmessung wird erwartet, daß der Methode ein kinetisches Meßprinzip zugrundeliegt und Probenfrequenz und Zuverlässigkeit der Methode den Anforderungen des Routinelaboratoriums genügen. Die bisher bekannten automatischen Bestimmungsmethoden der Pseudocholinesterase erfüllen nicht gleichzeitig diese Bedingungen. JENSEN-Holm (5) gibt eine Bestimmungsmethode der Cholinesterase an, die auf der automatischen Titration der freigesetzten Essigsäure bei konstantem $\mathrm{pH}$ beruht. Das Verfahren erreicht eine hohe Präzision
(VK 1,5\%); da die Proben jedoch nur einzeln gemessen werden können, ist sein praktischer Wert beschränkt. Andere automatische Bestimmungsverfahren der Pseudocholinesterase verwenden das Autoanalyzerprinzip (6-9), wobei einige Autoren ebenfalls Acetylthiocholin als Substrat und 5:5'-Dithiobis-2-nitrobenzosäure als SH-Gruppen-Reagenz verwenden. Das Meßprinzip des Autoanalyzers ist jedoch kein kinetisches, und es haften diesen Verfahren alle Nachteile' einer Zweipunktmessung an. Bei der vorliegenden Methode wird die Aktivität der Pseudocholinesterase kinetisch gemessen. Die Analysenfrequenz beträgt $120 / \mathrm{h}$, Präzision und Richtigkeit der Methode erfüllen die Zuverlässigkeitskriterien der Qualitätskontrolle. Das Verfahren kann für die Anwendung im Routinebetrieb empfohlen werden.

\section{Literatur}

1. Ellman, G. L., Courtney, D. K., Andres jr., V. \& FeatherSTONE, R. M. (1961), Biochem. Pharmacol. 7, 88-95. - 2. Garry, P. J. \& Routh, J. I. (1965), Clin. Chem. 11, 91-96. 3. Weber, H. \& Richterich, R. (1963), Klin. Wochenschr. 41, 665-667. - 4. Levine, J. B. (1970), Methoden der enzymatischen Analyse (BERgmeYER, H. U.) Hrsg.: Bd. I, Seite 813-815 Verlag Chemie, Weinheim/Bergstraße, 2. Auflage. - 5. Jensen-Holm, J.,
Lausen, H. H., Milthers, K. \&. Molteer, K. O. (1959), Acta Pharmacol. Toxicol. 15, 384-394. - 6. JAQUEs LongPre, A. R. T. (1971), Can. J. Tech. 33, 213-217. - 7. Boutin, D. \& BRodeur, J. (1,970), Clin. Biochem. 3, 245-254. - 8. Boutrn, D. \& BroDEUR, J. (1970), Clin. Biochem. 3, 3-9. - 9. Baum, G., Ward, F. B. \& Yaverbaum, S. (1972), Clin. Chim. Acta 36, 405-408.

Dr. A. Eberhard und Dr. R. Kley Zentrallabor der Klinischen Anstalten der RWTH Aachen

51 Aachen

Goethestraße 27/29 